Asian Journal of Information Technology 11 (1): 1-6, 2012

ISSN: $1682-3915$

(C) Medwell Journals, 2012

\title{
Design Strategy of Global e-School in Learning Ecology for Global-Citizenship
}

\author{
Kee-Burm Park \\ Seoul National University of Education, Woomyoun-Street 161, Seocho-Gu, 132-742 Seoul, Korea
}

\begin{abstract}
Today, countries are exchanging by own needs. For the first time, globalization has been in progress for market orientation. However, the globalization of market orientation causes many problems because of the lack of knowledge for cultural specificity in different areas. This study focuses on making a Global e-School (GeS) for global citizen education. I tried to make GeS by adopting ecology. When building a GeS, comparing and applying with ecology is needed, emergent learning by entering into a relation with specific culture based members, coevolution of a study community which creates opulent learning environments that consist of strong intelligence and understanding and acceptance of mutual culture in lens of multicultural education. GeS has a mission to develop qualities of global citizens in the global society based digital network environment.
\end{abstract}

Key words: Learning ecology, Global e-School, global-citizenship, network, global society, Korea

\section{INTRODUCTION}

Today, many countries find themselves in a variety of complex relationships. Socioeconomically countries depend on each other for various natural and human resources. Issues arising within a particular country may not just affect its particular region but also the entire world. The effects of demonstrations carried out in Southeast Asian countries are not confined to just that country but are felt by people around the world.

Southeast Asian countries which need oil are interested in a chaotic state of the Middle East. The workforce has experienced globalization. The amount of Asians that must move for economic reasons is increasing. Many foreigners enter Korea for more profitable employment every year. The diversity in their backgrounds and cultures, means they bring a multitude of different ways of life and viewpoints to Korea.

These days the definition of community is radically changing. During the 20th century the largest level of community was the national community. This has broadened to include the global community. Global community is produced by the interaction of politics, economics and cultures. This interplay is called the global ecosystem. Problems which occur in a particular nation must often be solved on the international level. These problems must be understood in the context of a complex cross network with a circular linking structure.

The effects of the global ecosystem are felt on the individual as well as the national level. Citizens of many nations are exposed to more political, economic and cultural influences than previously. Diversity of global culture is the emergent power for the development of global communities and has positive effects. Diversity is the seed of development. Respect for variety and difference is based on trust gained through interaction. Furthermore, exchange and interchange catalyzes the emergence of the global community and often results in mutual interdependence. This makes the global ecosystem virtuous and circular, guaranteeing sustainability. Circular Binterchange by needs $>$ understanding one another $>$ being intensified interchange $>$ self-development of global ecosystem can continue constantly.

Online schools provide the opportunity for more variety and depth in the learning experience. This study aims at making a strategy for comprehensive understanding of the global ecosystem.

\section{GeS LEARNING ECOSYSTEM}

Needs of GeS from ecological point of view: Ecological explanations give meaning ful implication to the learning community by exchanging information, creating new knowledge and replacing the old knowledge with the new. According to previous mechanistic dualism, truth is out of existence and humans can explore the truth. Mechanistic dualism says that humans make the point clear by knowing objective truth. This results in reductive thinking when applied to specific situations. After all they tend to simplify the world. However, this mechanistic dualism and decontextual thinking oversimplifies situations. Its error is to exclude their inherent characteristics by cutting off branches from situational context in various time and spatial gaps.

Removing inherent characteristics from any situation causes errors in comprehension and makes it hard to solve problems. Because the subject is always more complex than the object, this makes problems. When 
researchers look at the big picture, mechanistic dualism causes the destruction of the environment and climatic change and a lack of understanding and prejudice about other cultures.

Ecological approach includes the context and is more comprehensive than mechanistic dualism. Members in any community share their identities which intimately connect with others existence. Moreover, they make huge ecologic communities. Reducing and changing of special regions affects other regions. This ecological approach comprehends complex systems today.

If researchers applied the ecological approach to comprehensive international societies, it would be informative. In international societies, all countries have their own identities and are respected. They are related to each other. The boundaries of education is over specific countries and societies and it makes humans need more qualities.

If instruction and learning overcomes boundaries of countries and increases knowledge, researchers help us understand larger ranges through ecological comparing.

Emergent learning through interchange of learning communities: Objects in the ecological system works by needs through this interchange comes emergent results. Global citizens are able to experience learning and process knowledge efficiently. They can expand learning prospects by creating knowledge of relationship or context which is linked in life (Wenger, 2005). If results of learning are individual knowledge, it would be results of interchange and negotiating with members and it could make emergent knowledge in the community in circular links.

Providing productive learning environments with cross pollination and coevolved possibilities of learning communities through consisting knowledge: Ecosystems coevolve and are affected by the interaction of species. In terms of ecosystem, various countries exist individually and have a net link structure. In terms of government, economy, society and culture and so on, aspects of communication improve this net link structures. Each country has a related system which cannot separated.

Unique learning communities in a global world can share various materials, ideas and processes of learning. The results of this process have better qualities. Crossing the boundary of a country, sharing and exchanging important factors of learning can explain cross pollination of the ecosystem. Cross pollination will make more productive learning. Also, the results of this learning can have stronger meaning. This means development and evolution of learning communities, this is the same as co-evolution within the ecosystem.
Comprehension and acceptance of inter cultures: The global network involves global learning communities that respond according to needs. However, these needs can create natural understanding about learning community's individual cultures.

Various comprehension of culture with interchange between global learning communities can overcome prejudices of which culture is superior or inferior. Moreover, a society of unique learning communities can generate a new culture's flow toward meaningful change.

\section{PRINCIPLE OF GeS ECOSYSTEM'S STRUCTURE}

If researchers apply the meaning of the ecosystem in the GeS plan, researchers need to explore general features of this ecosystem. The general features are as follows (Yun and Chae, 2005).

First, the ecosystem consists of various species. The relationships between each species are linked by a complex food chain.

Second, energy flows in from outside of the ecosystem continually. This means energy is flowing and materials are circulating through a food chain. Transferred energy releases a type of thermal energy. The flow of energy is irreversible in the ecosystem. Each step higher, total energy decreases and efficiency of energy increases. Third, the ecosystem is coevolving. Ecosystems and species affect each other and evolve interdependently. For example, some lizards have vital poison. But some snakes which eat these lizards are immune to this poison. If some species of lizards had poison by chance, they would live well. Their numbers would increase. At the same time, snakes which are immune to this poison would also increase because of securing more food.

Fourth, the ecosystem maintains it's homeostasis through auto-regulation. However, the ecosystem resources are limited if this limits are surpassed, the ecosystem will be destroyed and extinction of species will occur. Basic principles of maintaining and developing ecosystem are as follows:

First, a principle called Interdependence (Capra, 1996; Han, 2002). It can be understood with relationship. Each objects' meanings are independent and clear. Furthermore, it works as a driving force to continuously renew oneself and the ecology that the one belongs to. Interdependence can prospect but it cannot prospect as embryological features. The whole is bigger than the sum of parts and emergence is a good expression for explaining the principle of interdependence. The second, principle is self-organization (Johnson, 2002; Yu and Chae, 2005). 
If a huge forest disappears in a lightening strike, it will grow again rapidly and become stabilized. Like this, the ecosystem wants to stabilize and keep balance according to stimulation of the ecosystem internally or externally. This explains the principles of selforganization. Lightening and forest fires were not expected before. In other words, researchers cannot expect the result of the relationship with lightening and life. We can call this emergence. Emergence means the whole is bigger than the sum of parts compared with reductive thinking total is same with the sum of parts. Finally, self-organization is operated by embryological theories in mechanism of relationship.

The third, principle is no increase, no decrease (Jeon, 2000; Yun and Chae, 2005). Ecosystems maintain and stabilize processeess of production application (sharing and consumption) extinction (substitution or modification) without outside controls. A reason that ecosystem's objects sometimes rapidly increase and stabilize again is a principle of no increase, no decrease.

The fourth, principle is no one, no two. A fruit and a seed is not one but researchers don't regard these separately. A fruit and a seed exist at the same time and relate to each other. It is not only not one but also not two in relationship (Lee, 2002). We can see that objects in the ecosystem have peculiar characteristics and relate to each other. Living with symbiosis and admitting difference in each object is a basic quality of being global citizens.

The fifth, principle is variety. Narrow breeding is pointed out by regression in an ecosystem. When objects of various characters relate to the each other and show off diversified color, the ecosystem will co-evolve well.

Today multicultural societies strive on diversity. because the diversity is a driving force in developing the community. Admitting difference makes the probability of more beauty and efficiency pushing the society higher.

\section{FACTORS OF GeS LEARNING ECOSYSTEM'S STRUCTURE}

Factors of GeS structure: Researchers can understand that an ecosystem is a living organism that maintains mutual relationships. An ecosystem does not operate by artificial external action, it exists on its own and keep its life. Creating, continuing and changing an ecosystem spontaneously based on necessity among its objects provides important points to build and develop a virtual global learning community. Specifically, the digital network internet comes into play as an instrument and environment for making links. The internet is also a cross pollinating mediator used to link various learning communities. Moreover, internet gives much information

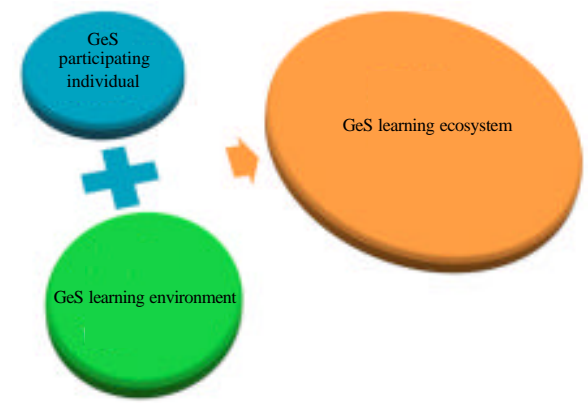

Fig. 1: The approach requisties for GeS learning ecosystem

and related knowledge and supports active energy. Information and knowledge that are in the learning ecosystem becomes new knowledge (Fig. 1). Internet based GeS (Global e-School) is accessed to something such as build-motive of GeS for evolving spontaneously. The first, GeS should be a place for spontaneous attendance.

Learning is interpreted as intellectual hunting in the learning ecosystem. Students can access GeS for good food (information) out of school. It is possible to have access to good information and learn in high quality. GeS is able to provide fresh global information. Good information or material is based on high level learning. The need of students is the motive to continue. Because $\mathrm{GeS}$ is not made in any institution like a normal learning community, the ecosystem should be biogenesis to accomplish interrelation and interactivity based on student's need.

The second, scaffolding is needed for smoothing interactivity in the GeS learning environment. According to the theory of Community of Practice, GeS is interpreted as meaning the boundary object. Therefore, various scaffolding systems should be built to be able to communicate fluently. Above all a mediator who can overcome language barriers is needed.

The third, GeS that guarantees variety and interactivity becomes the basis of an equal society. Based on the above statements, GeS should be an open community which introduces needed information and knowledge freely and enables sharing each other. A society or a country has cultural backgrounds and ideas which separates it from others. Important things, inspection and exclusion about specific knowledge should reach a minimum.

Establishing relations with other individuals and communities start with a plural vision. This makes positive changes and strong students by increasing Heterosis. Various interactivity requires respect from all students. Prejudice and injustice towards others is a fatal activity that can collapse the whole of ecosystem. 
The fourth, GeS imports external energy then it gives students sufficient access to information. Members who attend learning sessions need creative environments to take on the roles of a producer. Much information and knowledge is shared in a process of dynamic activity among members. Sharing knowledge through the process of interactivity can make students producer of knowledge and real prosumers while identifying themselves.

\section{FACTORS OF DETAILED FUNCTION}

Based on the strategy of building the GeS learning ecosystem, factors of detailed functions for designing a model of GeS learning ecosystem as shown in Fig. 2.

First of all if the GeS learning ecosystem is to be maintained continuosly. It must match with the demands of students. Especially when the learning situation which is needed by students builds pertinently. Students will attend the GeS learning ecosystem.

A global virtual school environment has an organic relation with some facts a scaffolding program, an open learning community, an environment of a prosumer and a reward system and it should help the adaptation and development process of students. In terms of assistance, a language arbitator to improve communication and learning contents to be produced in one's mother tongue must be offered. The meaning of open learning communities enact attributes of the ecosystem and participants which are in various cultures communicate freely. Creating an environment for a prosumer is a process which makes information and knowledge available through interanction besides basic contents. based on mother-tongue. A reward system is related with the GeS learning ecosystem's possibility of continuity. This provides external motivation which leads to quantitative and qualitative development with proper rewards.

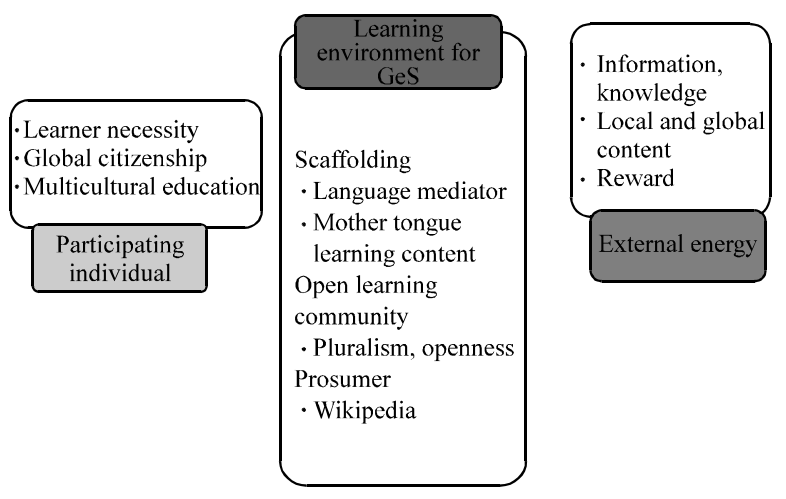

Fig. 2: The subtle-ties for GeS learning ecosystem

\section{STRATEGIES TO BUILD GeS LEARNING ECOSYSTEM}

Based on the ecological figure of speech for build a learning ecosystem, strategies for building the GeS learning ecosystem are as follows. First, in order that students can attend as members of a learning ecosystem spontaneously, GeS should meet the needs of students.

Second, scaffolding is needed in order that students can attend and communicate. Specially, it has to have language mediators. The language mediators that can speak over two languages help interaction among students in GeS. Also, they can guide learning activities as virtual teachers. The language mediators function as active mediators in elementary schools. They also are able to be guiders of interaction.

Furthermore, GeS provides basic learning contents based on one's mother tongue. It helps students to catch up on basic knowledge. Providing learning contents based on one's mother tongue contributes to realizing equal education at the global level. The third, GeS should be an open place that guarantees openness and pluralism. The GeS learning ecosystem can be interpreted as a variety of information and the supply of knowledge as inflow of energy. Providing various information and knowledge as energy supply is possible through a learning ecosystem with openness and pluralism.

GeS based on various cultures guarantees the higher pluralism which is more than members of attending communities. The purpose of GeS education is to respect each other and to internationalize the quality as global citizens through various cultural and thinking exchanges. Therefore, the mission statement of GeS must be established and announced after consideration and consultation with the communities which make up global countries.

Fourth, for the purpose of establishing common goals which are needed for the success of GeS, collecting and sharing information which will help achieve the common goals. Researchers should build a global system which produces collective intelligence. We should support a chain process which is made by cooperative activities based on discussion. Also, we select and arrange activities which are related to various information and knowledge based on the living location's civilization and nature in environment. Students who attend $\mathrm{GeS}$ are prosumers of information. Students can be producers and consumers of information in the GeS learning ecosystem. Consumption, interaction and production of information is circularly achieved by using communication tools of interaction such as BBS (Bulletin Board System), chatting 


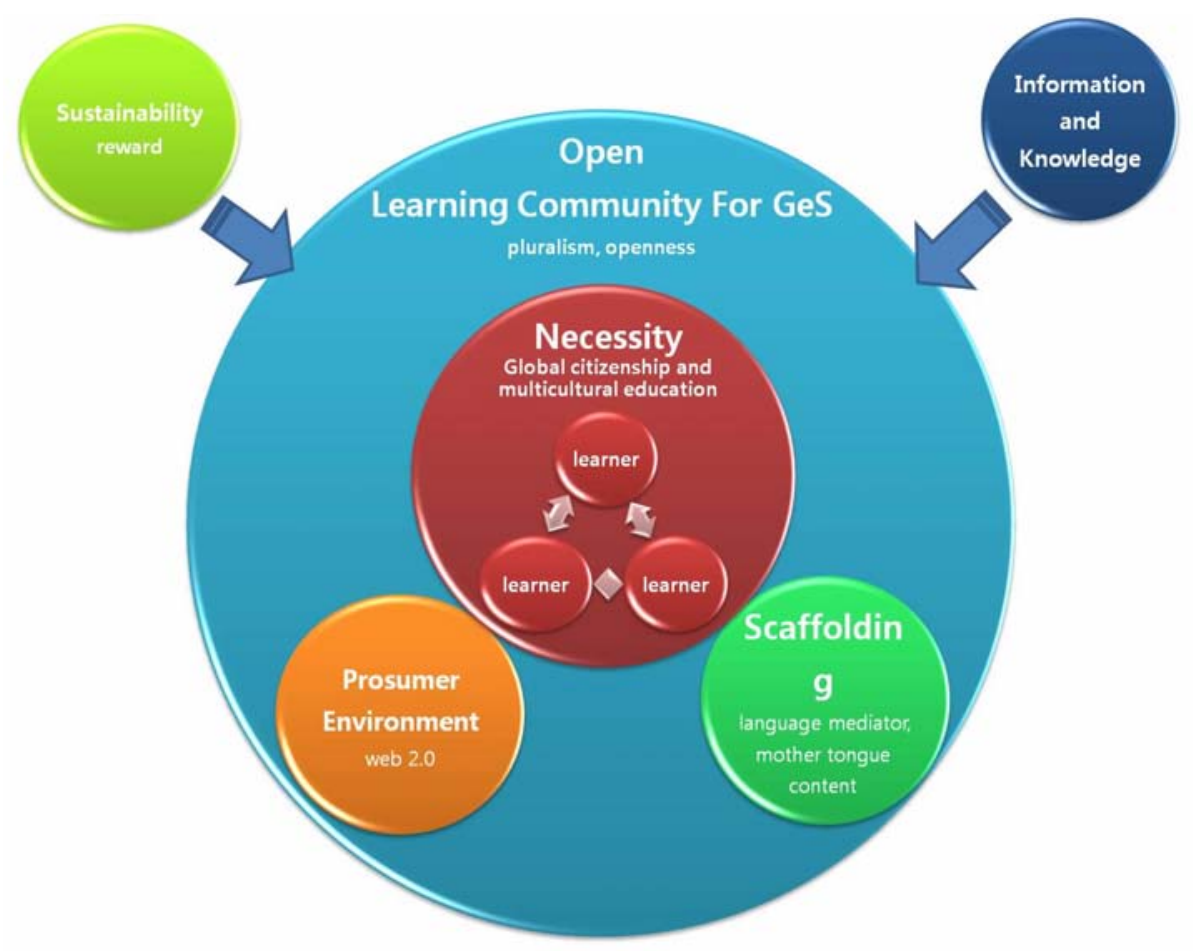

Fig. 3: GeS learning ecosystem

and E-mail. Also, high quality information and collective intelligence produced by an interaction are selected and used by other members. The GeS learning ecosystem is correspondent to a Web2.0 which not only develops given information into new information but also makes new information.

Fifth, the system should keep continuing GeS's sustainable possibility by giving participants rewards. It has to offer proper rewards to individuals or communities that do projects which produce information of high quality. The examples of proper rewards are formal rewards like certificates of merit published by a principal or licenses and qualification for student exchange. If the rewards are effective then healthy participants will attend the learning ecosystem continuously, optimize the learning ecosystem and develop a climax forest. Structuralizing system of the GeS learning ecosystem based on the GeS building strategy and functional element is Fig. 3.

Finally, the GeS system consist of objects that participate by necessity, they adapt and develop the learning ecosystem through interaction with other learning environments and members. Information and knowledge which are created in various locations as external energy will be energy which supports a durable and dynamic learning ecosystem.

\section{CONCLUSION}

Global e-School (GeS) is for global citizen education based on understanding among cultures. This study shows the ecological theory needed to build the GeS. Building the GeS through ecological analogy and application is needed for several reasons such as emergent learning through interchange among various members based on different cultures, making productive learning environments, coevolution of learning communities and understanding and acceptance between different cultures in various cultural education. These are foundation stones for world citizen education.

The strategy to build GeS consists of three parts. First, in the view of students. Students should meet their needs for participation as a member of a learning ecosystem.

Any system which is attending and communicating with the GeS must have scaffolding devices like a language arbitrator and should be an open space that guarantees openness and pluralism. Also, in the view of the prosumer, researchers have to build a common goal and gather information to achieve it. A system which produces common knowledge should be built.

Last, GeS should retain sustainability as a learning ecosystem by offering rewards to high level participants. 
The needs of students and the GeS's environments must continue as a steady-state learning ecosystem with supply and demand of information and knowledge.

GeS aims to develop the quality of citizens into world citizens based on high digital network environments. If the GeS doesn't fit the role of object of a boundary which coexists between various cultures and consists of a learning system prescribed concepts and culture, GeS will not continue long. GeS induces basic concepts such as ability of self-management, possibility of continuation, climax forest and mutual relation. It should be built as a growing place of learning, an ecosystem itself. Although, $\mathrm{GeS}$ starts based on requests by students in its initial phases, students that operate and attend an original curriculum can develop requirements and qualifications for admission in a learning community. Each will contribute to the attendance of stabilizing mutual relations in the ecosystem.

The reasons for opening, attending, sharing and communicating in a digital network society aren't made based on hypothetical possibilities. It is made necessary by requests and eager desires.

\section{REFERENCES}

Capra, F., 1996. The Web of Life: The New Scientific Understanding of Living System. Anchor Books, New York, USA., ISBN: 9780385476768, Pages: 397.

Han, S.H., 2002. A Lifelong Learning and Learning Ecosystem. Hakjisa Publisher, Seoul, South Korea.

Jeon, K.S., 2000. The Culturology of Cultural Era. Iljisa, Seoul.

Johnson, S., 2002. Emergence: The Connected Lives of Ants, Brains, Citges and Software. Touchstone, New York.

Lee, D.H., 2002. A Synthesize of Ecology and Wha-Jaeng Idea. The Professor Newspaper Minumsa, Seoul.

Wenger, E., 2005. Communities of Practice: Learning Meaning and Identity. Cambridge University Press, Nwe York, USA.

Yun, Y.S. and S.B. Chae, 2005. An introduction to complex systems. Samsung Economic Research Institute, Seoul. 